\title{
SERUM ADENOSINE DEAMINASE ACTIVITY IN MONITORING DISEASE ACTIVITY AND RESPONSE TO THERAPY IN SEVERE PSORIASIS
}

\begin{abstract}
Zülal Erbagci ${ }^{1}$, A. Binnur Erbagci ${ }^{2}$, Oya Köylüoglu ${ }^{2}$, A. Almila Tuncel ${ }^{1}$
Gaziantep University, Medical Faculty, Gaziantep, Turkey: Department of Dermatology ${ }^{1}$, Biochemistry and Clinical Biochemistry $^{2}$

Summary: Objectives: Activity of Serum Adenosine Deaminase (ADA), a main enzyme in purine degradation and considered as a marker for non-specific $\mathrm{T}$ cell activation, in psoriasis has been investigated in a few studies with conflicting results. Design and Methods: To evaluate the significance of serum ADA activity in psoriasis, and analyze whether ADA activity may be related to disease activity, we performed a prospective study with 38 cases of psoriasis and 24 healthy volunteers. Patients were divided into two groups as cases with local and stable lesions (Group i, n: 20) and severe cases with extensive involvement (Group ii, n: 18). Serum ADA activity was determined by modified Guisti procedure. Results: When taken into consideration of all patients -regardless of the severity of the disease- the mean serum ADA activity of psoriatics did not differ significantly from that of controls $(p>0.05)$. However, it was higher in Group ii than in Group i and healthy controls (respectively $p<0.001$ and $p<0.05$ ). A significant decrease was observed also after therapy only in Group ii $(p<0.001)$. Conclusion: Serum ADA activity may be correlated to the disease activity of severe psoriasis. We suggest that it might be a serologic marker for follow-up of in such cases. It could be used in predicting relapses before clinical findings as well as in deciding to stop or decrease systemic therapies at the right time, which have potential to cause severe systemic side effects when given for a long period. Further studies with larger case populations are required to support our findings.
\end{abstract}

Key words: Psoriasis; Severity; Disease activity; Serum adenosine deaminase activity

\section{Introduction}

Adenosine Deaminase (ADA; EC 3.5.4.4), catalyses the irreversible hydrolytic deamination of adenosine and deoxyadenosine to yield inosine and deoxyinosine, respectively, as a part of purine salvage pathway. It contributes to regulation of intracellular and extracellular concentrations of adenosine along with 5 ' nucleotidase and adenosine kinase. ADA, which is widely distributed in most mammalian tissues, exhibits the highest activity in lymphoid tissues, and is essential for the proliferation, maturation and function of lymphocytes, particularly those of T cell lineage. ADA activity has been considered as a marker of non-specific T-cell activation $(1,20)$.

Psoriasis is a common, chronic and inflammatory skin disorder, characterized by a variable clinical course. Although the exact etiology of psoriasis is unknown yet, genetic predisposition, infections, traumas, metabolic and endocrine alterations, stress and certain drugs have been implicated. Psoriasis is currently considered as an immunological disorder because of its associations with certain human leukocyte-associated antigens, spontaneous exacerbations and remissions of disease, the presence of activated $\mathrm{T}$ lymphocytes in lesions, and good responses to immuno- suppressive therapies (3). Activated T lymphocytes and their cytokine products seem to have important roles in the formation and extension of the skin lesions. Elevated ADA activity has already been shown in the involved psoriatic epidermis $(9,11,18)$. However, this may simply reflect accelerated salvage pathway of nucleic acid metabolism associated with the hyperproliferative status of epidermis in psoriasis. Therefore measurement of ADA activity in serum might be more significant in determining importance of immunological factors in the pathogenesis of psoriasis. Nevertheless, conflicting results have been reported from previous studies $(5,10,11)$.

We conducted a prospective clinical and biochemical case-control study to investigate serum ADA activity in patients with psoriasis of various types and severity, and its relationship with disease activity and response to therapy.

\section{Patients and Methods}

\section{Subjects}

Thirty eight patients with psoriasis referring to University of Gaziantep, Medical Faculty, Department of Dermatology and 24 healthy volunteers, without clinical, laboratory or histological evidence of any disease, were included in the 
study, after giving informed consent. Subjects receiving any systemic or topical therapy within the last month preceding the study or having an established diagnosis of any systemic disease with either clinical or laboratory signs were excluded. The permission of institutional Review Board was obtained before the start of the study.

According to the subtype or extension of the disease, cases with psoriasis were divided into two groups as cases with local and stable lesions (Group i, n: 20) and severe cases with extensive involvement (Group ii, n: 18). Baseline characteristics of the participants are presented in Table1. All patients were re-analyzed for serum ADA activity after complete improvement, regardless of the kind and duration of the therapy protocol.

\section{Experimental Study}

Blood samples were collected using standard venipuncture technique between 9:30 to 11:00 am after $12 \mathrm{~h}$ fasting. Serum samples were separated immediately after centrifugation at $4^{\circ} \mathrm{C}, 2000 \mathrm{~g}$ for $10 \mathrm{~min}$ and stored at $-20^{\circ} \mathrm{C}$ until analysis. Serum total ADA activity was determined at $37{ }^{\circ} \mathrm{C}$ according to the method of Giusti and Galanti (6) based on the Bertholet reaction, i.e. the formation of coloured indophenol complexes from ammonia liberated from adenosine, quantified spectrophotometrically. A serum pool was used as a presicion control and was included in each analytical run. Intraassay and interassay precision performances of the assay were determined on 10 replicates in a single run and in 20 different runs respectively, and yielded coefficients of variation (CV) $2.8 \%$ and $4.2 \%$ respectively at $16 \mathrm{U} / \mathrm{L}$ level. One unit of ADA is defined as the amount of enzyme required to release $1 \mu \mathrm{mol} / \mathrm{min}$ of ammonia from adenosine at standard assay conditions.

\section{Statistical Analyses}

Data were presented as mean \pm SD or lower and upper ranges, and median. Comparison of variables was performed by Student's $t$-test or Mann Whitney- $U$ test when necessary. Case-control differences in nominal data were evaluated with the $X^{2}$ test. Two tailed $\mathrm{p}$ values $<0.05$ were considered significant. Finally, power analysis was performed to see present effect sizes. SPSS 9.0 (Statistical Packages for Social Sciences inc., Chicago, illinois, USA) and Power And Precision $^{\mathrm{TM}}$ (Biostat, inc., Englewood, NJ 07631, USA) programs were used for statistical analyses and illustrations.

\section{Results}

The study group of thirty-eight patients with psoriasis was composed of 18 women and 20 males with a mean age $( \pm \mathrm{SD})$ of $33.6 \pm 14.4$ years, range $15-80$ years. The control group consisted of 11 female and 13 male subjects with a mean age of $35.9 \pm 15.1$ years, range 14-73 years. No statistically significant difference was observed in age and gender distributions between patients and controls as well as between the cases in Group i and those in Group ii ( $p>0.05$ ). However duration of the disease in Group ii was significantly shorter than in Group i $(\mathrm{p}<0.05)$ (Table 1$)$. In Group i, plaque type lesions were limited on the knees and elbows in 9 of patients, scalp in 5, palms in 2, palms and soles in 1, and various body sites in 3 patients. Treatment modalities used in this group consisted of topical corticosteroids plus anthralin in 10 patients, topical corticosteroids combined with $0.005 \%$ calcipotriol and 5\% salicylic acid in 6, UVB phototherapy in 1 and local PUVA therapy in 3 patients. In Group ii, 6 cases had generalized plaque type psoriasis, 7 had erythrodermic psoriasis and the remaining 5 had generalized pustular psoriasis. Clearance of the lesions in this group was obtained with PUVA in 4 patients, ReUVB (acitretin plus UVB) in 1, RePUVA (acitretin plus PUVA) in 7 , acitretin alone in 4, methotrexate in 1, and cyclosporine in 1 patient. Four of the cases with generalized plaque type psoriasis were treated with acitretin (mean duration of therapy: 8 months, limits: 6-10 months), and 2 with PUVA

Tab. 1: Demographic data of patient groups and therapies used in cases.

\begin{tabular}{|l|l|l|}
\hline & \multicolumn{1}{|c|}{ Group i (n: 20) } & \multicolumn{1}{|c|}{ Group ii (n: 18) } \\
\hline Gender & F: 9, M: 11 & F: 9 M: 9 \\
\hline Age (years) & 16-80 (Median: 28.5) & 5-61 (Median: 34.5) \\
\hline Disease duration & 5 to 25 years & 3 months to 4 years \\
\hline Family history & Positive: 13; Negative: 7 & Positive: 12; Negative: 6 \\
\hline Localisation/Type of psoriasis & Knee-elbow: 9 & Generalized plaques: 6 \\
& Scalp: 5 & Erythrodermic: 7 \\
& Palmar: 2 & Pustular: 5 \\
& Plantar: 1 & \\
& Variable: 3 & \\
\hline Treatments & Topical corticosteroids + Anthralin: 10 & PUVA: 4 \\
& T.corticosteroids + Psorcutan + & Re-UVB: 1 \\
& Salicylic acid: 6 & Re-PUVA: 7 \\
& UVB: 1 & Asitretin: 4 \\
\hline & Lokal (palmar/plantar) PUVA: 3 & Metothrexate: 1 \\
\hline & & Cyclosporin: 1 \\
\hline
\end{tabular}


for 4 and 6 months. Clearance of the erythrodermic psoriasis was obtained in 1 case with ReUVB for 8 months, in 2 cases with PUVA for 6 and 7 months, in 2 case with RePUVA for 5 and 6 months, and in each of the remaining two cases with methotrexate for 6 weeks and with cyclosporine for 4 weeks. Cases with generalized pustular psoriasis were treated with RePUVA for a mean of 4.4 months (limits: 3-7 months).

Mean $( \pm$ SD) serum ADA activity of the patients was $15.05 \pm 6.8 \mathrm{U} / \mathrm{L}$ and slightly higher than that of control group (14.5 $\pm 2.45 \mathrm{U} / \mathrm{L})$, but difference was not statistically significant $(p>0.05)$. For the present effect size of the patient and the control groups'ADA activities (population means of 15.05 versus $14.5 \mathrm{U} / \mathrm{L}$, SD 6.8 vs $2.45 \mathrm{U} / \mathrm{L}$, sample size 38 and 24, and alpha 0.05-two tailed) power was 0.066. Mean serum ADA activities in Group i and Group ii were respectively $11.3 \pm 2.8 \mathrm{U} / \mathrm{L}$ (median: $14 \mathrm{U} / \mathrm{L}$, min-max:

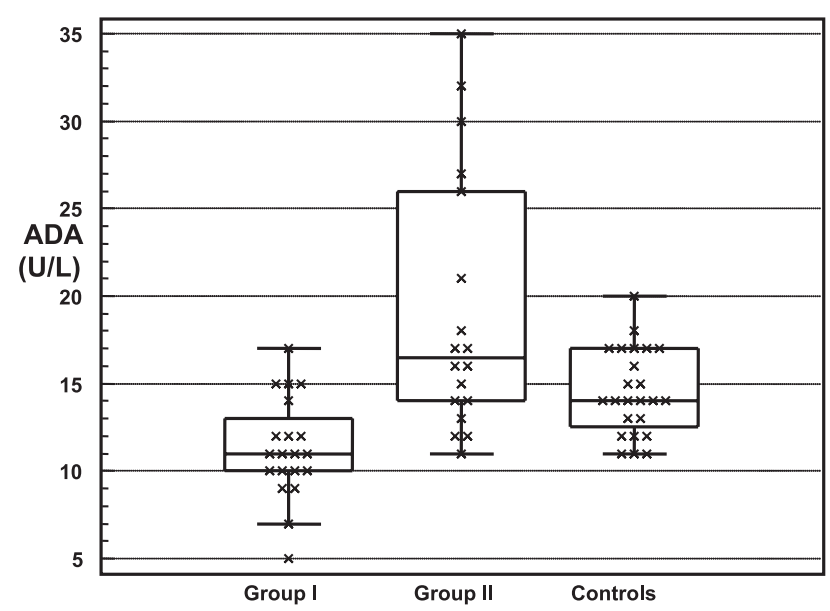

Fig. 1: Serum ADA levels in psoriasis subgroups and controls.

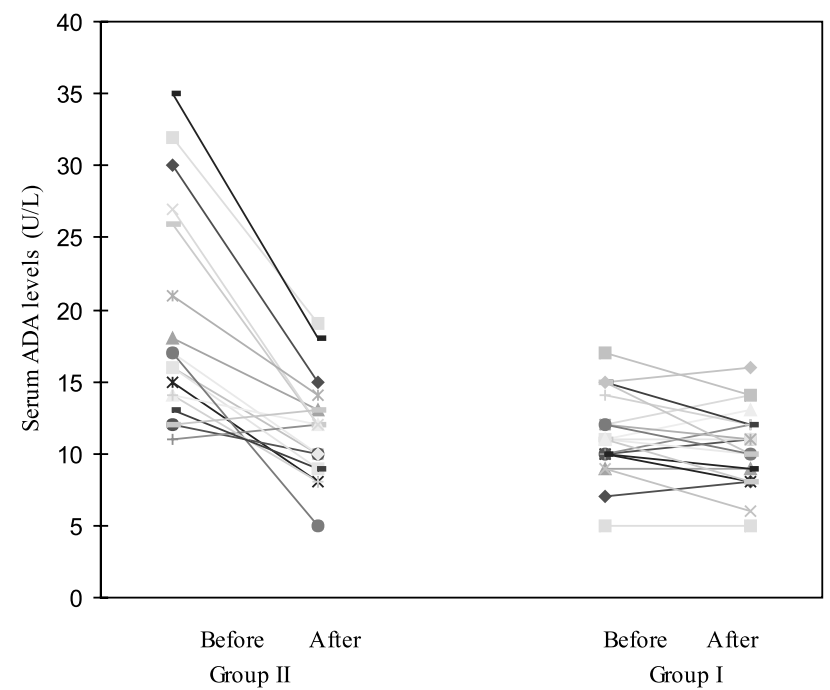

Fig. 2: Pre- and post-treatment serum ADA levels in Group ii and Group i psoriasis.
11-20 U/L) and 19.36 \pm 7.48 (median: $16.5 \mathrm{U} / \mathrm{L}$, min-max: 11-35 U/L). The mean serum ADA activity of Group ii was significantly higher than that of Group i $[\mathrm{p}<0.001$, power: 0.99 (population means 19.36 vs 11.3 , SD 7.48 vs 2.8 , sample sizes 18 and 20, alpha 0.05-two tailed)]. Also, it was higher than that of healthy controls $[p<0.05$, power: 0.83 (population means 19.36 vs 14.5 , SD 7.48 vs 2.45 , sample sizes 18 and 24, alpha 0.05-two tailed)]. These results mean that $99 \%$ and $83 \%$ of studies would be expected to yield a significant effect (Figure 1).

A significant decrease was observed in the mean ADA activity in Group ii after treatment (mean: $11.59 \mathrm{U} / \mathrm{L}$, median: $12 \mathrm{U} / \mathrm{L}$, min-max: 5-19 U/L, $\mathrm{p}<0.001$ ). For the present effect size of Group ii pre- and after-treatment ADA activities [population mean $( \pm \mathrm{SD})$ difference of $7.6 \pm 5.5$ U/L (95\% Ci: 4.9-10.2 U/L), sample size 18, and alpha 0.05 -two tailed] power was 1.000 meaning that close to $100 \%$ of studies would be expected to yield a significant effect. No significant decrease was found in the mean ADA activity of Group i after treatment (mean: $10.45 \mathrm{U} / \mathrm{L}$, median: $10.5 \mathrm{U} / \mathrm{L}$, min-max: $5-16 \mathrm{U} / \mathrm{L})(\mathrm{p}>0.05$, power: 0.438 ) [Population mean $( \pm \mathrm{SD})$ difference of $0.85 \pm 2.00 \mathrm{U} / \mathrm{L}$ (95\% Ci: 0.069-1.769 U/L), sample size 20, and alpha 0.05-two tailed]. (Figure 2).

\section{Discussion}

The number of proliferating keratinocytes has been reported to be more than 10 -fold greater in involved psoriatic skin compared to those of uninvolved skin of psoriatics and normal skin of healthy subjects (20). Tikhonov et al (18) have observed decreased purine nucleotide mono-phosphates, increased purine nucleotide degradation end-products and two-fold increased ADA and purine nucleoside phosphorylase in the skin and erythrocytes of patients with psoriasis. A decrease in the activity of cyclic adenosine monophosphate (cAMP) cascade, which is an inhibitor of cell proliferation, may explain increased cellular proliferation in psoriatic epidermis. Since adenosine, the substrate of ADA, stimulates cAMP cascade in the epidermis, ADA might have an important role in the regulation of keratinocyte proliferation through cAMP levels $(9,20)$. ADA activity has been found constantly elevated in psoriatic epidermis compared to uninvolved skin $(9-11,18)$. Nevertheless, data on the systemic ADA activity, i.e. In sera, lymphocytes and erythrocytes, in psoriasis are conflicting.

Iizuka et al (7) found normal ADA activity in peripheral blood lymphocytes in patients with psoriasis. Koizumi and Ohkawara (10) also observed no significant increases in serum ADA activities in 18 psoriatic patients. Beckman et al (4) found no difference between erythrocyte ADA levels in psoriatics and normal controls as well. However, Vlcek and Mikulikova (19) reported higher ADA activities in peripheral blood lymphocytes from 32 patients with psoriasis compared to controls. Moreover, they observed significant decreases in ADA activities after treatment with 
methotrexate. Bükülmez et al (5) found significantly elevated ADA levels in 25 patients compared to 15 healthy subjects as well as significant decreases in the ADA activities in 10 patients after treatment with either PUVA or cyclosporine compared to pretreatment values, suggesting a strong relationship between T cell activation and ADA activity. Lack of correlation with PASi scores and ADA activity in the mentioned study was explained with PASi being a subjective method and not sensitive in reflecting disease activity. Most recently, Köse et al (11) evaluated 30 patients with psoriasis and observed that mean plasma ADA activity was within laboratory reference limits whereas they noted statistically significantly higher baseline plasma ADA activities in a subgroup consisting of 20 patients compared to those in 15 healthy controls. They observed also significant increases in tissue ADA activity and decreases in erythrocyte ADA activity in patients before treatment, and opposite results after therapy with propylthiouracil that has antiproliferative and immunomodulatory effects.

Elevated serum ADA activity has been observed in lymphoid malignancies $(7,17)$, infectious and non-infectious systemic diseases with chronic $\mathrm{T}$ cell activation such as pulmonary and pleural tuberculosis $(8,16)$, sarcoidosis (2), typhoid fever (14), and connective tissue diseases including progressive systemic sclerosis, morphea, and dermatomyositis $(13,15)$. This information constituted our exclusion criteria for both patients and controls and all subjects were evaluated before the study regarding any systemic diseases by clinical and laboratory tests.

In the present study, psoriasis activity was not monitored with PASi scores because there has been significant differences in inter- and intra-observer variability in PASi scoring, and necessity for more objective methods has been emphasized in previous reports $(5,12)$. Therefore complete clearance of the lesions was required for post-treatment evaluation. When taken into consideration of all patients - regardless of the severity of the disease - we could not found any significant difference between serum ADA activities in psoriatics and controls. Further studies with larger case populations are needed to make this matter clear. However, on the basis of our results, we have demonstrated that elevated serum ADA activity is correlated with severe psoriasis with extensive involvement, reflecting the role of $\mathrm{T}$ cell activation in the pathogenesis of psoriasis of grave course. Moreover, significant decreases in serum ADA levels were observed after therapy in this patient group.

In conclusion, serum ADA activity may be correlated to the disease activity of severe psoriasis. We suggest that it might be a serologic marker for follow-up of active and progressive disease. A decrease in serum ADA activity in such cases could be considered as a predictor of clinical improvement and transferring to inactive course as well. Determination of serum ADA level may help in deciding to stop or to decrease systemic therapies at the right time that may cause severe systemic side effects when used for a long period, or conversely, it may be used in predicting relapses before development of clinical findings. Nevertheless further studies with larger case populations are required to support our suggestions.

\section{References}

1. Adams A, Harkness RA. Adenosine deaminase activity in thymus and other human tissues. Clin Exp immunol 1976;26:647-9.

2. Albera C, Mabritto i, Ghio P, Solidoro P, Marchetti L, Pozzi E. Adenosine deaminase activity and fibronectin levels in bronchoalveolar lavage fluid in sarcoidosis and tuberculosis. Sarcoidosis 1993;10:18-25.

3. Baker BS, Fry L. The immunology of psoriasis. Br J Dermatol 1992;126:1-9.

4. Beckman G, Beckman L, Cedergren B, Liden S. Red cell enzyme types in psoriasis. Hum Hered 1977;27:408-11.

5. Bükülmez G, Akan T, Ciliv G. Serum adenosine deaminase levels in patients with psoriasis: a prospective case-control study. Eur J Dermatol. 2000;10:274-6.

6. Giusti G, Galanti B. Colorimetric method. In: Methods of Enzymatic Analysis (Bergmeyer HU, ed.). Weinheim: Verlag Chemie, 1984:315-23.

7. Iizuka H, Koizumi H, Kimura T, Miura Y. Adenosine deaminase activity in peripheral lymphocytes of psoriasis and Sézary's syndrome. J Dermatol 1980;7 $165-9$.

8. Kayacan O, Karnak D, Delibalta M, Beder S, Karaca L, Tutkak H. Adenosine deaminase activity in bronchoalveolar lavage in Turkish patients with smear negative pulmonary tuberculosis. Respir Med 2002;96:536-41.

9. Koizumi H, iizuka H, Aoyagi T, Miura Y. Adenosine deaminase in human epidermis from healthy and psoriatic subjects. Arch Dermatol Res 1983;275:340-4.

10. Koizumi H, Ohkawara A. Adenosine deaminase activity in sera of patients with psoriasis, mycosis fungoides and adult $\mathrm{T}$ cell leukemia. Acta Derm Venereol (Stockh) 1992;72:410-12.

11. Köse K, Utas S, Yazici C, Akdas A, Kelestimur F. Effect of propylthiouracil on adenosine deaminase activity and thyroid function in patients with psoriasis. $\mathrm{Br}$ J Dermatol 2001;144:1121-6.

12. Marks R, Barton SP, Shuttleworth D, Finlay AY. Assessment of disease progress in psoriasis. Arch Dermatol 1989;125:235-40.

13. Meunier P, Filipe P, Emerit i, Freitas J, Guerra Rodrigo F, Manso C. Adenosine deaminase in progressive systemic sclerosis. Acta Derm Venereol. 1995;75:297-9.

14. Nardiello S, Russo M, Pizzella T, Galanti B. Selectively increased adenosine deaminase levels in T cells during typhoid fever. J Clin Lab immunol 1984;14 103-5.

15. Sasaki T, Nakajima H. Serum adenosine deaminase activity in systemic sclerosis (scleroderma) and related disorders. J Am Acad Dermatol 1992;27:411-4

16. Shibagaki T, Hasegawa Y, Saito H, Yamori S, Shimokata K. Adenosine deaminase isozymes in tuberculous pleural effusion. J Lab Clin Med 1996;127:348-52.

17. Simpkins H, Stanton A, Davis BH. Adenosine deaminase activity in lymphoid subpopulations and leukemias. Cancer Res 1981;41:3107-10.

18. Tikhonov Yu V, Markusheva Li, Toguzov RT. Metabolism of purine compounds in psoriasis. Klin Lab Diagn 1998;3:3-6.

19. Vlcek F, Mikulikova D. The effect of methotrexate on activity of T-lymphocyte marker enzymes in patients with psoriasis vulgaris. Bratisl Lek Listy 1995;96: $137-40$

20. Woorhees JJ. Commentary: cyclic adenosine monophosphate regulation of normal and psoriatic epidermis. Arch Dermatol 1982;118:869-74.

Submitted February 2006.

Accepted May 2006.

Prof. Zülal Erbagci, M.D.,

Gaziantep University, Medical Faculty, Gazimuhtarpasa Bulvari, Gecit 1. No: 1/5, 27090 Gaziantep, Turkey. e-mail: zerbagci@yahoo.com 